\title{
The Rhetorical Parsing of Natural Language Texts
}

\author{
Daniel Marcu \\ Department of Computer Science \\ University of Toronto \\ Toronto, Ontario \\ Canada M5S 3G4 \\ marcuecs . toronto edu
}

\begin{abstract}
We derive the rhetorical structures of texts by means of two new, surface-form-based algorithms: one that identifies discourse usages of cue phrases and breaks sentences into clauses, and one that produces valid rhetorical structure trees for unrestricted natural language texts. The algorithms use information that was derived from a corpus analysis of cue phrases.
\end{abstract}

\section{Introduction}

Researchers of natural language have repeatedly acknowledged that texts are not just a sequence of words nor even a sequence of clauses and sentences. However, despite the impressive number of discourse-related theories that have been proposed so far, there have emerged no algorithms capable of deriving the discourse structure of an unrestricted text. On one hand, efforts such as those described by Asher (1993), Lascarides, Asher, and Oberlander (1992), Kamp and Reyle (1993), Grover et al. (1994), and Prüst, Scha, and van den Berg (1994) take the position that discourse structures can be built only in conjunction with fully specified clause and sentence structures. And Hobbs's theory (1990) assumes that sophisticated knowledge bases and inference mechanisms are needed for determining the relations between discourse units. Despite the formal elegance of these approaches, they are very domain dependent and, therefore, unable to handle more than a few restricted examples. On the other hand, although the theories described by Grosz and Sidner (1986), Polanyi (1988), and Mann and Thompson (1988) are successfully applied manually, they are too informal to support an automatic approach to discourse analysis.

In contrast with this previous work, the rhetorical parser that we present builds discourse trees for unrestricted texts. We first discuss the key concepts on which our approach relies (section 2) and the corpus analysis (section 3) that provides the empirical data for our rhetorical parsing algorithm. We discuss then an algorithm that recognizes discourse usages of cue phrases and that determines clause boundaries within sentences. Lastly, we present the rhetorical parser and an example of its operation (section 4).

\section{Foundation}

The mathematical foundations of the rhetorical parsing algorithm rely on a first-order formalization of valid text structures (Marcu, 1997). The assumptions of the formalization are the following. 1. The elementary units of complex text structures are non-overlapping spans of text. 2. Rhetorical, coherence, and cohesive relations hold between textual units of various sizes. 3. Relations can be partitioned into two classes: paratactic and hypotactic. Paratactic relations are those that hold between spans of equal importance. Hypotactic relations are those that hold between a span that is essential for the writer's purpose, i.e., a nucleus, and a span that increases the understanding of the nucleus but is not essential for the writer's purpose, i.e., a satellite. 4. The abstract structure of most texts is a binary, tree-like structure. 5 . If a relation holds between two textual spans of the tree structure of a text, that relation also holds between the most important units of the constituent subspans. The most important units of a textual span are determined recursively: they correspond to the most important units of the immediate subspans when the relation that holds between these subspans is paratactic, and to the most important units of the nucleus subspan when the relation that holds between the immediate subspans is hypotactic.

In our previous work (Marcu, 1996), we presented a complete axiomatization of these principles in the context of Rhetorical Structure Theory (Mann and Thompson, 1988) and we described an algorithm that, starting from the set of textual units that make up a text and the set of elementary rhetorical relations that hold between these units, can derive all the valid discourse trees of that text. Consequently, if one is to build discourse trees for unrestricted texts, the problems that remain to be solved are the automatic determination of the textual units and the rhetorical relations that hold between them. In this paper, we show how one can find and exploit approximate solutions for both of these problems by capitalizing on the occurrences of certain lexicogrammatical constructs. Such constructs can include tense 
and aspect (Moens and Steedman, 1988; Webber, 1988; Lascarides and Asher, 1993), certain patterns of pronominalization and anaphoric usages (Sidner, 1981; Grosz and Sidner, 1986; Sumita et al., 1992; Grosz, Joshi, and Weinstein, 1995), it-clefts (Delin and Oberlander, 1992), and discourse markers or cue phrases (Ballard, Conrad, and Longacre, 1971; Halliday and Hasan, 1976; Van Dijk, 1979; Longacre, 1983; Grosz and Sidner, 1986; Schiffrin, 1987; Cohen, 1987; Redeker, 1990; Sanders, Spooren, and Noordman, 1992; Hirschberg and Litman, 1993; Knott, 1995; Fraser, 1996; Moser and Moore, 1997). In the work described here, we investigate how far we can get by focusing our attention only on discourse markers and lexicogrammatical constructs that can be detected by a shallow analysis of natural language texts.

The intuition behind our choice relies on the following facts:

- Psycholinguistic and other empirical research (Kintsch, 1977; Schiffrin, 1987; Segal, Duchan, and Scott, 1991; Cahn, 1992; Sanders, Spooren, and Noordman, 1992; Hirschberg and Litman, 1993; Knott, 1995; Costermans and Fayol, 1997) has shown that discourse markers are consistently used by human subjects both as cohesive ties between adjacent clauses and as "macroconnectors" between larger textual units. Therefore, we can use them as rhetorical indicators at any of the following levels: clause, sentence, paragraph, and text.

- The number of discourse markers in a typical text - approximately one marker for every two clauses (Redeker, 1990) - is sufficiently large to enable the derivation of rich rhetorical structures for texts.

- Discourse markers are used in a manner that is consistent with the semantics and pragmatics of the discourse segments that they relate. In other words, we assume that the texts that we process are well-formed from a discourse perspective, much as researchers in sentence parsing assume that they are well-formed from a syntactic perspective. As a consequence, we assume that one can bootstrap the full syntactic, semantic, and pragmatic analysis of the clauses that make up a text and still end up with a reliable discourse structure for that text.

Given the above discussion, the immediate objection that one can raise is that discourse markers are doubly ambiguous: in some cases, their use is only sentential, i.e., they make a semantic contribution to the interpretation of a clause; and even in the cases where markers have a discourse usage, they are ambiguous with respect to the rhetorical relations that they mark and the sizes of the textual spans that they connect. We address now each of these objections in turn.

Sentential and discourse usages of cue phrases. Empirical studies on the disambiguation of cue phrases (Hirschberg and Litman, 1993) have shown that just by considering the orthographic environment in which a discourse marker occurs, one can distinguish between sentential and discourse usages in about $80 \%$ of cases. We have taken Hirschberg and Litman's research one step further and designed a comprehensive corpus analysis that enabled us to improve their results and coverage. The method, procedure, and results of our corpus analysis are discussed in section 3 .

Discourse markers are ambiguous with respect to the rhetorical relations that they mark and the sizes of the units that they connect. When we began this research, no empirical data supported the extent to which this ambiguity characterizes natural language texts. To better understand this problem, the corpus analysis described in section 3 was designed so as to also provide information about the types of rhetorical relations, rhetorical statuses (nucleus or satellite), and sizes of textual spans that each marker can indicate. We knew from the beginning that it would be impossible to predict exactly the types of relations and the sizes of the spans that a given cue marks. However, given that the structure that we are trying to build is highly constrained, such a prediction proved to be unnecessary: the overall constraints on the structure of discourse that we enumerated in the beginning of this section cancel out most of the configurations of elementary constraints that do not yield correct discourse trees.

Consider, for example, the following text:

(1) [Although discourse markers are ambiguous, ${ }^{1}$ ] [one can use them to build discourse trees for unrestricted texts: ${ }^{2}$ ] [this will lead to many new applications in natural language processing. ${ }^{3}$ ]

For the sake of the argument, assume that we are able to break text (1) into textual units as labelled above and that we are interested now in finding rhetorical relations between these units. Assume now that we can infer that Although marks a CONCESSIVE relation between satellite 1 and nucleus either 2 or 3 , and the colon, an ELABORATION between satellite 3 and nucleus either 1 or 2 . If we use the convention that hypotactic relations are represented as first-order predicates having the form rhet_rel(NAME, satellite, nucleus) and that paratactic relations are represented as predicates having the form rhet_rel(NAME, nucleus $s_{1}$, nucleus $s_{2}$ ), a correct representation for text (1) is then the set of two disjunctions given in (2):

$$
\left\{\begin{array}{c}
\text { rhet rel(CONCESSION, 1, 2) } \vee \\
\text { rhet_rel(CONCESSION, 1, 3) } \\
\text { rhet rel(ELABORATION, 3, 1) } \vee \\
\text { rhet_rel(ELABORATION, 3, 2) }
\end{array}\right.
$$

Despite the ambiguity of the relations, the overall rhetorical structure constraints will associate only one discourse tree with text (1), namely the tree given in figure 1: any discourse tree configuration that uses relations rhet $\operatorname{rel}($ CONCESSION , 1,3) and rhet rel(ELABORATION, 3,1) will be ruled out. For example, relation rhet rel(ELABORATION, 3, 1) will be ruled 


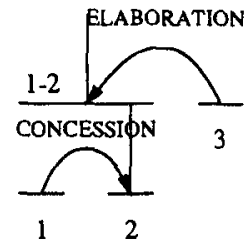

Figure 1: The discourse tree of text (1).

out because unit 1 is not an important unit for span $[1,2]$ and, as mentioned at the beginning of this section, a rhetorical relation that holds between two spans of a valid text structure must also hold between their most important units: the important unit of span $[1,2]$ is unit 2, i.e., the nucleus of the relation rhet rel(CONCESSION, 1,2).

\section{A corpus analysis of discourse markers}

\subsection{Materials}

We used previous work on cue phrases (Halliday and Hasan, 1976; Grosz and Sidner, 1986; Martin, 1992; Hirschberg and Litman, 1993; Knott, 1995; Fraser, 1996) to create an initial set of more than 450 potential discourse markers. For each potential discourse marker, we then used an automatic procedure that extracted from the Brown corpus a set of text fragments. Each text fragment contained a "window" of approximately 200 words and an emphasized occurrence of a marker. On average, we randomly selected approximately 19 text fragments per marker, having few texts for the markers that do not occur very often in the corpus and up to 60 text fragments for markers such as and, which we considered to be highly ambiguous. Overall, we randomly selected more than 7900 texts.

All the text fragments associated with a potential cue phrase were paired with a set of slots in which an analyst described the following. 1. The orthographic environment that characterizes the usage of the potential discourse marker. This included occurrences of periods, commas, colons, semicolons, etc. 2. The type of usage: Sentential, Discourse, or Both. 3. The position of the marker in the textual unit to which it belonged: Beginning, Medial, or End. 4. The right boundary of the textual unit associated with the marker. 5 . The relative position of the textual unit that the unit containing the marker was connected to: Before or After. 6. The rhetorical relations that the cue phrase signaled. 7. The textual types of the units connected by the discourse marker: from Clause to Multiple Paragraph. 8. The rhetorical status of each textual unit involved in the relation: Nucleus or Satellite. The algorithms described in this paper rely on the results derived from the analysis of 1600 of the 7900 text fragments.

\subsection{Procedure}

After the slots for each text fragment were filled, the results were automatically exported into a relational database. The database was then examined semiautomatically with the purpose of deriving procedures that a shallow analyzer could use to identify discourse usages of cue phrases, break sentences into clauses, and hypothesize rhetorical relations between textual units. For each discourse usage of a cue phrase, we derived the following:

- A regular expression that contains an unambiguous cue phrase instantiation and its orthographic environment. A cue phrase is assigned a regular expression if, in the corpus, it has a discourse usage in most of its occurrences and if a shallow analyzer can detect it and the boundaries of the textual units that it connects. For example, the regular expression "[,] although " identifies such a discourse usage.

- A procedure that can be used by a shallow analyzer to determine the boundaries of the textual unit to which the cue phrase belongs. For example, the procedure associated with "[,] although " instructs the analyzer that the textual unit that pertains to this cue phrase starts at the marker and ends at the end of the sentence or at a position to be determined by the procedure associated with the subsequent discourse marker that occurs in that sentence.

- A procedure that can be used by a shallow analyzer to hypothesize the sizes of the textual units that the cue phrase relates and the rhetorical relations that may hold between these units. For example, the procedure associated with "[,] although " will hypothesize that there exists a CONCESSION between the clause to which it belongs and the clause(s) that went before in the same sentence. For most markers this procedure makes disjunctive hypotheses of the kind shown in (2) above.

\subsection{Results}

At the time of writing, we have identified 1253 occurrences of cue phrases that exhibit discourse usages and associated with each of them procedures that instruct a shallow analyzer how the surrounding text should be broken into textual units. This information is used by an algorithm that concurrently identifies discourse usages of cue phrases and determines the clauses that a text is made of. The algorithm examines a text sentence by sentence and determines a set of potential discourse markers that occur in each sentence. It then applies left to right the procedures that are associated with each potential marker. These procedures have the following possible effects:

- They can cause an immediate breaking of the current sentence into clauses. For example, when an "[.] although " marker is found, a new clause, whose right boundary is just before the occurrence of the marker, is created. The algorithm is then recursively applied on the text that is found 


\begin{tabular}{|l|c|c|c|c|c|}
\hline Text & $\begin{array}{c}\text { No. of discourse } \\
\text { markers identified } \\
\text { manually }\end{array}$ & $\begin{array}{c}\text { No. of discourse } \\
\text { markers identified } \\
\text { by the algorithm }\end{array}$ & $\begin{array}{c}\text { No. of discourse } \\
\text { markers identified } \\
\text { correctly } \\
\text { by the algorithm }\end{array}$ & Recall & Precision \\
\hline 1. & 174 & 169 & 150 & $86.2 \%$ & $88.8 \%$ \\
\hline 2. & 63 & 55 & 49 & $77.8 \%$ & $89.1 \%$ \\
\hline 3. & 38 & 24 & 23 & $63.2 \%$ & $95.6 \%$ \\
\hline Total & 275 & 248 & 222 & $80.8 \%$ & $89.5 \%$ \\
\hline
\end{tabular}

Table 1: Evaluation of the marker identification procedure.

\begin{tabular}{|l|c|c|c|c|c|c|}
\hline Text & $\begin{array}{c}\text { No. of } \\
\text { sentences }\end{array}$ & $\begin{array}{c}\text { No. of clause } \\
\text { boundaries identified } \\
\text { manually }\end{array}$ & $\begin{array}{c}\text { No. of clause } \\
\text { boundaries identified } \\
\text { by the algorithm }\end{array}$ & $\begin{array}{c}\text { No. of clause } \\
\text { boundaries identified } \\
\text { correctly } \\
\text { by the algorithm }\end{array}$ & Recall & Precision \\
\hline 1. & 242 & 428 & 416 & 371 & $86.7 \%$ & $89.2 \%$ \\
\hline 2. & 80 & 151 & 123 & 113 & $74.8 \%$ & $91.8 \%$ \\
\hline 3. & 19 & 61 & 37 & 36 & $59.0 \%$ & $97.3 \%$ \\
\hline Total & 341 & 640 & 576 & 520 & $81.3 \%$ & $90.3 \%$ \\
\hline
\end{tabular}

Table 2: Evaluation of the clause boundary identification procedure.

between the occurrence of "[,] although" and the end of the sentence.

- They can cause the setting of a flag. For example, when an "Although" marker is found, a flag is set to instruct the analyzer to break the current sentence at the first occurrence of a comma.

- They can cause a cue phrase to be identified as having a discourse usage. For example, when the cue phrase "Although" is identified, it is also assigned a discourse usage. The decision of whether a cue phrase is considered to have a discourse usage is sometimes based on the context in which that phrase occurs, i.e., it depends on the occurrence of other cue phrases. For example, an "and" will not be assigned a discourse usage in most of the cases; however, when it occurs in conjunction with "although", i.e., "and although", it will be assigned such a role.

The most important criterion for using a cue phrase in the marker identification procedure is that the cue phrase (together with its orthographic neighborhood) is used as a discourse marker in at least $90 \%$ of the examples that were extracted from the corpus. The enforcement of this criterion reduces on one hand the recall of the discourse markers that can be detected, but on the other hand, increases significantly the precision. We chose this deliberately because, during the corpus analysis, we noticed that most of the markers that connect large textual units can be identified by a shallow analyzer. In fact, the discourse marker that is responsible for most of our algorithm recall failures is and. Since a shallow analyzer cannot identify with sufficient precision whether an occurrence of and has a discourse or a sentential usage, most of its occurrences are therefore ignored. It is true that, in this way, the discourse structures that we build lose some potential finer granularity, but fortunately, from a rhetorical analysis perspective, the loss has insignificant global repercussions: the vast majority of the relations that we miss due to recall failures of and are JOINT and SEQUENCE relations that hold between adjacent clauses.

Evaluation. To evaluate our algorithm, we randomly selected three texts, each belonging to a different genre:

1. an expository text of 5036 words from Scientific American;

2. a magazine article of $\mathbf{1 5 8 8}$ words from Time;

3. a narration of 583 words from the Brown Corpus.

Three independent judges, graduate students in computational linguistics, broke the texts into clauses. The judges were given no instructions about the criteria that they had to apply in order to determine the clause boundaries; rather, they were supposed to rely on their intuition and preferred definition of clause. The locations in texts that were labelled as clause boundaries by at least two of the three judges were considered to be "valid clause boundaries". We used the valid clause boundaries assigned by judges as indicators of discourse usages of cue phrases and we determined manually the cue phrases that signalled a discourse relation. For example, if an "and" was used in a sentence and if the judges agreed that a clause boundary existed just before the "and", we assigned that "and" a discourse usage. Otherwise, we assigned it a sentential usage. Hence, we manually determined all discourse usages of cue phrases and all discourse boundaries between elementary units.

We then applied our marker and clause identification algorithm on the same texts. Our algorithm found $80.8 \%$ of the discourse markers with a precision of $89.5 \%$ (see 
INPUT: a text $T$.

1. Determine the set $D$ of all discourse markers and the set $U_{T}$ of elementary textual units in $T$.

2. Hypothesize a set of relations $R$ between the elements of $U_{T}$.

3. Use a constraint satisfaction procedure to determine all the discourse trees of $T$.

4. Assign a weight to each of the discourse trees and determine the tree(s) with maximal weight.

Figure 2: Outline of the rhetorical parsing algorithm

table 1), a result that outperforms Hirschberg and Litman's (1993). The same algorithm identified correctly $81.3 \%$ of the clause boundaries, with a precision of $90.3 \%$ (see table 2). We are not aware of any surface-form-based algorithms that achieve similar results.

\section{Building up discourse trees}

\subsection{The rhetorical parsing algorithm}

The rhetorical parsing algorithm is outlined in figure 2 . In the first step, the marker and clause identification algorithm is applied. Once the textual units are determined, the rhetorical parser uses the procedures derived from the corpus analysis to hypothesize rhetorical relations between the textual units. A constraint-satisfaction procedure similar to that described in (Marcu, 1996) then determines all the valid discourse trees (see (Marcu, 1997) for details). The rhetorical parsing algorithm has been fully implemented in $\mathrm{C}++$.

Discourse is ambiguous the same way sentences are: more than one discourse structure is usually produced for a text. In our experiments, we noticed, at least for English, that the "best" discourse trees are usually those that are skewed to the right. We believe that the explanation of this observation is that text processing is, essentially, a left-to-right process. Usually, people write texts so that the most important ideas go first, both at the paragraph and at the text level. ${ }^{1}$ The more text writers add, the more they elaborate on the text that went before: as a consequence, incremental discourse building consists mostly of expansion of the right branches. In order to deal with the ambiguity of discourse, the rhetorical parser computes a weight for each valid discourse tree and retains only those that are maximal. The weight function reflects how skewed to the right a tree is.

\subsection{The rhetorical parser in operation}

Consider the following text from the November 1996 issue of Scientific American (3). The words in italics denote the discourse markers, the square brackets denote

\footnotetext{
'In fact, journalists are trained to employ this "pyramid" approach to writing consciously (Cumming and McKercher, 1994).
}

the boundaries of elementary textual units, and the curly brackets denote the boundaries of parenthetical textual units that were determined by the rhetorical parser (see Marcu (1997) for details); the numbers associated with the square brackets are identification labels.

(3) [With its distant orbit $1-50$ percent farther from the sun than Earth - Jand slim atmospheric blanket, ${ }^{1}$ ] [Mars experiences frigid weather conditions. ${ }^{2}$ ] [Surface temperatures typically average about -60 degrees Celsius $(-76$ degrees Fahrenheit) at the equator and can dip to -123 degrees $C$ near the poles. ${ }^{3}$ ] [Only the midday sun at tropical latitudes is warm enough to thaw ice on occasion, ${ }^{4}$ [ [but any liquid water formed in this way would evaporate almost instantly ${ }^{5}$ [ because of the low atmospheric pressure. $^{6}$ ]

[Although the atmosphere holds a small amount of water, and water-ice clouds sometimes develop, ${ }^{7}$ ] [most Martian weather involves blowing dust or carbon dioxide. ${ }^{8}$ ] [Each winter, for example, a blizzard of frozen carbon dioxide rages over one pole, and a few meters of this dryice snow accumulate as previously frozen carbon dioxide evaporates from the opposite polar cap. $\left.{ }^{9}\right]$ [Yet even on the summer pole, \{where the sun remains in the sky all day long, \} temperatures never warm enough to melt frozen water. ${ }^{10}$ ]

Since parenthetical information is related only to the elementary unit that it belongs to, we do not assign it an elementary textual unit status. Such an assignment will only create problems at the formal level as well, because then discourse structures can no longer be represented as binary trees.

On the basis of the data derived from the corpus analysis, the algorithm hypothesizes the following set of relations between the textual units:

(4)

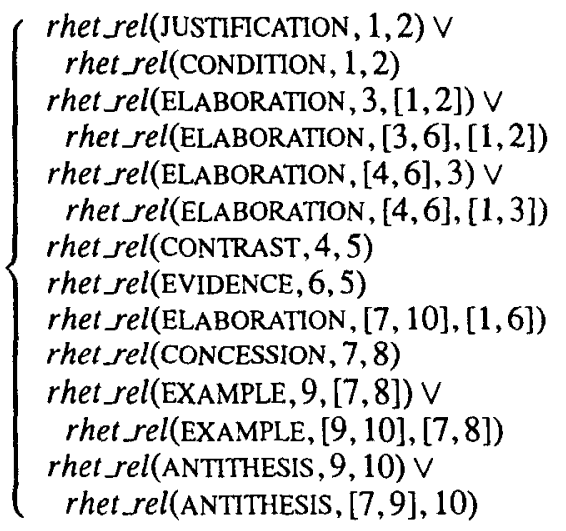

The algorithm then determines all the valid discourse trees that can be built for elementary units 1 to 10 , given the constraints in (4). In this case, the algorithm constructs 8 different trees. The trees are ordered according to their weights. The "best" tree for text (3) has weight 3 and is fully represented in figure 3. The PostScript file corresponding to figure 3 was automatically generated by 


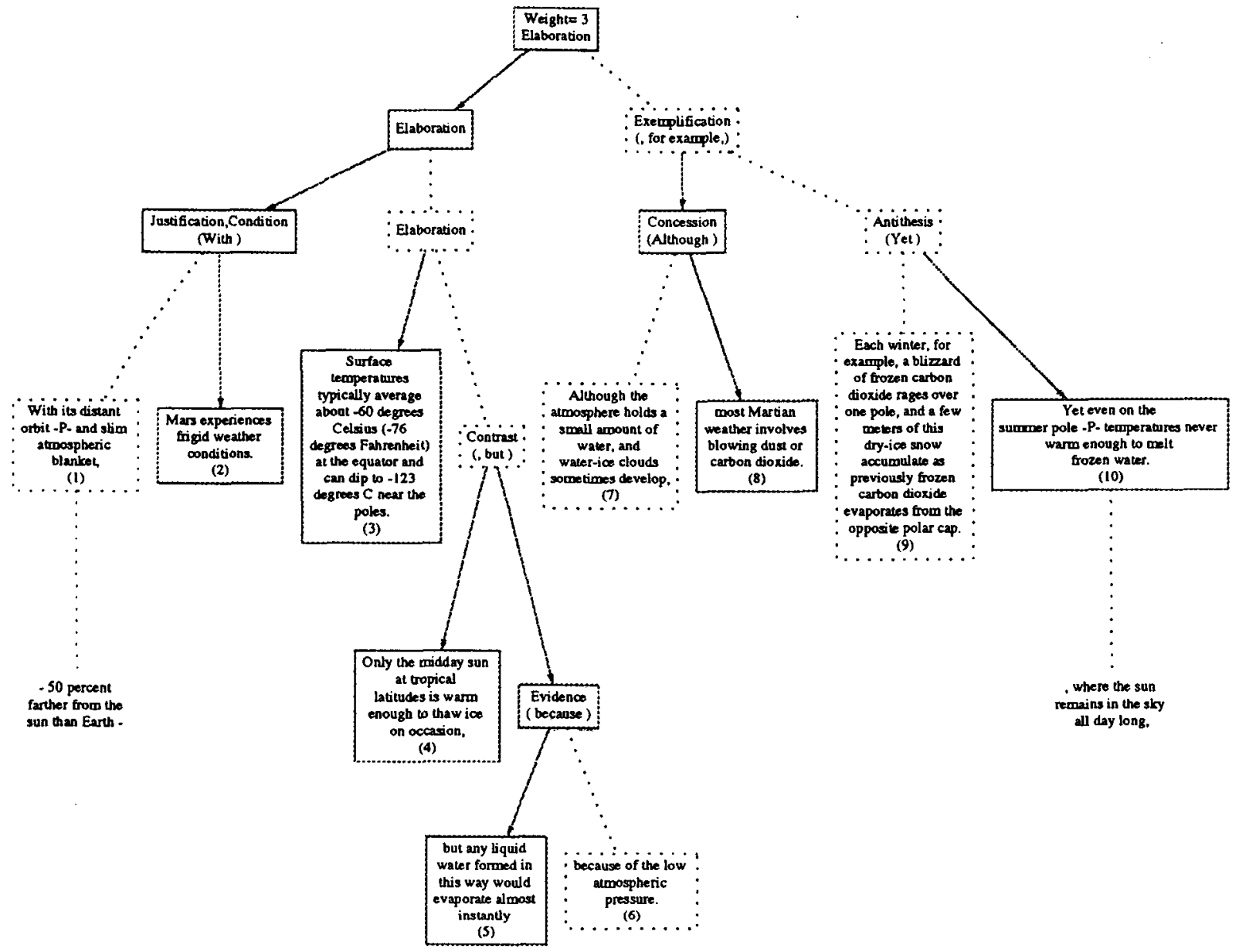

Figure 3: The discourse tree of maximal weight that can be associated with text (3).

a back-end algorithm that uses "dot", a preprocessor for drawing directed graphs. The convention that we use is that nuclei are surrounded by solid boxes and satellites by dotted boxes; the links between a node and the subordinate nucleus or nuclei are represented by solid arrows, and the links between a node and the subordinate satellites by dotted lines. The occurrences of parenthetical information are marked in the text by $\mathrm{a}-P$ - and a unique subordinate satellite that contains the parenthetical information.

\subsection{Discussion and evaluation}

We believe that there are two ways to evaluate the correctness of the discourse trees that an automatic process builds. One way is to compare the automatically derived trees with trees that have been built manually. Another way is to evaluate the impact that the discourse trees that we derive automatically have on the accuracy of other natural language processing tasks, such as anaphora resolution, intention recognition, or text summarization. In this paper, we describe evaluations that follow both these avenues.

Unfortunately, the linguistic community has not yet built a corpus of discourse trees against which our rhetorical parser can be evaluated with the effectiveness that traditional parsers are. To circumvent this problem, two analysts manually built the discourse trees for five texts that ranged from 161 to 725 words. Although there were some differences with respect to the names of the relations that the analysts used, the agreement with respect to the status assigned to various units (nuclei and satellites) and the overall shapes of the trees was significant.

In order to measure this agreement we associated an importance score to each textual unit in a tree and computed the Spearman correlation coefficients between the importance scores derived from the discourse trees built by each analyst. ${ }^{2}$ The Spearman correlation coefficient

\footnotetext{
${ }^{2}$ The Spearman rank correlation coefficient is an alternative to the usual correlation coefficient. It is based on the ranks of the data, and not on the data itself, and so is resistant to outliers. The null hypothesis tested by Spearman is that two variables
} 
between the ranks assigned for each textual unit on the bases of the discourse trees built by the two analysts was very high: 0.798 , at $p<0.0001$ level of significance. The differences between the two analysts came mainly from their interpretations of two of the texts: the discourse trees of one analyst mirrored the paragraph structure of the texts, while the discourse trees of the other mirrored a logical organization of the text, which that analyst believed to be important.

The Spearman correlation coefficients with respect to the importance of textual units between the discourse trees built by our program and those built by each analyst were $0.480, p<0.0001$ and $0.449, p<0.0001$. These lower correlation values were due to the differences in the overall shape of the trees and to the fact that the granularity of the discourse trees built by the program was not as fine as that of the trees built by the analysts.

Besides directly comparing the trees built by the program with those built by analysts, we also evaluated the impact that our trees could have on the task of summarizing text. A summarization program that uses the rhetorical parser described here recalled $66 \%$ of the sentences considered important by 13 judges in the same five texts, with a precision of $68 \%$. In contrast, a random procedure recalled, on average, only $38.4 \%$ of the sentences considered important by the judges, with a precision of $38.4 \%$. And the Microsoft Office 97 summarizer recalled $41 \%$ of the important sentences with a precision of $39 \%$. We discuss at length the experiments from which the data presented above was derived in (Marcu, 1997).

The rhetorical parser presented in this paper uses only the structural constraints that were enumerated in section 2. Co-relational constraints, focus, theme, anaphoric links, and other syntactic, semantic, and pragmatic factors do not yet play a role in our system, but we nevertheless expect them to reduce the number of valid discourse trees that can be associated with a text. We also expect that other robust methods for determining coherence relations between textual units, such as those described by Harabagiu and Moldovan (1995), will improve the accuracy of the routines that hypothesize the rhetorical relations that hold between adjacent units.

We are not aware of the existence of any other rhetorical parser for English. However, Sumita et al. (1992) report on a discourse analyzer for Japanese. Even if one ignores some computational "bonuses" that can be easily exploited by a Japanese discourse analyzer (such as co-reference and topic identification), there are still some key differences between Sumita's work and ours. Particularly important is the fact that the theoretical foundations of Sumita et al.'s analyzer do not seem to be able to accommodate the ambiguity of discourse markers: in their

are independent of each other, against the alternative hypothesis that the rank of a variable is correlated with the rank of another variable. The value of the statistic ranges from -1 , indicating that high ranks of one variable occur with low ranks of the other variable, through 0 , indicating no correlation between the variables, to +1 , indicating that high ranks of one variable occur with high ranks of the other variable. system, discourse markers are considered unambiguous with respect to the relations that they signal. In contrast, our system uses a mathematical model in which this ambiguity is acknowledged and appropriately treated. Also, the discourse trees that we build are very constrained structures (see section 2): as a consequence, we do not overgenerate invalid trees as Sumita et al. do. Furthermore, we use only surface-based methods for determining the markers and textual units and use clauses as the minimal units of the discourse trees. In contrast, Sumita et al. use deep syntactic and semantic processing techniques for determining the markers and the textual units and use sentences as minimal units in the discourse structures that they build. A detailed comparison of our work with Sumita et al.'s and others' work is given in (Marcu, 1997).

\section{Conclusion}

We introduced the notion of rhetorical parsing, i.e., the process through which natural language texts are automatically mapped into discourse trees. In order to make rhetorical parsing work, we improved previous algorithms for cue phrase disambiguation, and proposed new algorithms for determining the elementary textual units and for computing the valid discourse trees of a text. The solution that we described is both general and robust.

Acknowledgements. This research would have not been possible without the help of Graeme Hirst; there are no right words to thank him for it. I am grateful to Melanie Baljko, Phil Edmonds, and Steve Green for their help with the corpus analysis. This research was supported by the Natural Sciences and Engineering Research Council of Canada.

\section{References}

Asher, Nicholas. 1993. Reference to Abstract Objects in Discourse. Kluwer Academic Publishers, Dordrecht.

Ballard, D. Lee, Robert Conrad, and Robert E. Longacre. 1971. The deep and surface grammar of interclausal relations. Foundations of language, 4:70-118.

Cahn, Janet. 1992. An investigation into the correlation of cue phrases, unfilled pauses and the structuring of spoken discourse. In Proceedings of the IRCS Workshop on Prosody in Natural Speech, pages 19-30.

Cohen, Robin. 1987. Analyzing the structure of argumentative discourse. Computational Linguistics, 13(12):11-24, January-June.

Costermans, Jean and Michel Fayol. 1997. Processing Interclausal Relationships. Studies in the Production and Comprehension of Text. Lawrence Erlbaum Associates, Publishers.

Cumming, Carmen and Catherine McKercher. 1994. The Canadian Reporter: News writing and reporting. Hartcourt Brace. 
Delin, Judy L. and Jon Oberlander. 1992. Aspectswitching and subordination: the role of $i t$-clefts in discourse. In Proceedings of the Fourteenth International Conference on Computational Linguistics (COLING92), pages 281-287, Nantes, France, August 23-28.

Fraser, Bruce. 1996. Pragmatic markers. Pragmatics, 6(2):167-190.

Grosz, Barbara J., Aravind K. Joshi, and Scott Weinstein. 1995. Centering: A framework for modeling the local coherence of discourse. Computational Linguistics, 21(2):203-226, June.

Grosz, Barbara J. and Candace L. Sidner. 1986. Attention, intentions, and the structure of discourse. Computational Linguistics, 12(3):175-204, July-September.

Grover, Claire, Chris Brew, Suresh Manandhar, and Marc Moens. 1994. Priority union and generalization in discourse grammars. In Proceedings of the 32nd Annual Meeting of the Association for ComputationalLinguistics ( $A C L-94$ ), pages 17-24, Las Cruces, June 27-30.

Halliday, Michael A.K. and Ruqaiya Hasan. 1976. Cohesion in English. Longman.

Harabagiu, Sanda M. and Dan I. Moldovan. 1995. A marker-propagation algorithm for text coherence. In Working Notes of the Workshop on Parallel Processing in Artificial Intelligence, pages 76-86, Montreal, Canada, August.

Hirschberg, Julia and Diane Litman. 1993. Empirical studies on the disambiguation of cue phrases. Computational Linguistics, 19(3):501-530.

Hobbs, Jerry R. 1990. Literature and Cognition. CSLI Lecture Notes Number 21.

Kamp, Hand and Uwe Reyle. 1993. From Discourse to Logic: Introduction to ModelTheoretic Semantics of Natural Language, Formal Logic and Discourse Representation Theory. Kluwer Academic Publishers, London, Boston, Dordrecht. Studies in Linguistics and Philosophy, Volume 42.

Kintsch, Walter. 1977. On comprehending stories. In Marcel Just and Patricia Carpenter, editors, Cognitive processes in comprehension. Erlbaum, Hillsdale, New Jersey.

Knott, Alistair. 1995. A Data-Driven Methodology for Motivating a Set of Coherence Relations. Ph.D. thesis, University of Edinburgh.

Lascarides, Alex and Nicholas Asher. 1993. Temporal interpretation, discourse relations, and common sense entailment. Linguistics and Philosophy, 16(5):437493.

Lascarides, Alex, Nicholas Asher, and Jon Oberlander. 1992. Inferring discourse relations in context. In Proceedings of the 30th Annual Meeting of the Association for Computational Linguistics ( $A C L-92$ ), pages 1-8.

Longacre, Robert E. 1983. The Grammar of Discourse. Plenum Press, New York.
Mann, William C. and Sandra A. Thompson. 1988. Rhetorical structure theory: Toward a functional theory of text organization. Text, 8(3):243-281.

Marcu, Daniel. 1996. Building up rhetorical structure trees. In Proceedings of the Thirteenth National Conference on Artificial Intelligence $(A A A I-96)$, volume 2 , pages 1069-1074, Portland, Oregon, August 4-8,.

Marcu, Daniel. 1997. The rhetorical parsing, summarization, and generation of natural language texts. Ph.D. thesis, Department of Computer Science, University of Toronto, Forthcoming.

Martin, James R. 1992. English Text. System and Structure. John Benjamin Publishing Company, Philadelphia/Amsterdam.

Moens, Marc and Mark Steedman. 1988. Temporal ontology and temporal reference. Computational Linguistics, 14(2):15-28.

Moser, Megan and Johanna D. Moore. 1997. On the correlation of cues with discourse structure: Results from a corpus study. Submitted for publication.

Polanyi, Livia. 1988. A formal model of the structure of discourse. Journal of Pragmatics, 12:601-638.

Prüst, H., R. Scha, and M. van den Berg. 1994. Discourse grammar and verb phrase anaphora. Linguistics and Philosophy, 17(3):261-327, June.

Redeker, Gisela. 1990. Ideational and pragmatic markers of discourse structure. Journal of Pragmatics, 14:367381.

Sanders, Ted J.M., Wilbert P.M. Spooren, and Leo G.M. Noordman. 1992. Toward a taxonomy of coherence relations. Discourse Processes, 15:1-35.

Schiffrin, Deborah. 1987. Discourse Markers. Cambridge University Press.

Segal, Erwin M., Judith F. Duchan, and Paula J. Scott. 1991. The role of interclausal connectives in narrative structuring: Evidence from adults' interpretations of simple stories. Discourse Processes, 14:27-54.

Sidner, Candace L. 1981. Focusing for interpretation of pronouns. Computational Linguistics, 7(4):217-231, October-December.

Sumita, K., K. Ono, T. Chino, T. Ukita, and S. Amano. 1992. A discourse structure analyzer for Japanese text. In Proceedings of the International Conference on Fifth Generation Computer Systems, volume 2, pages 1133-1140.

Van Dijk, Teun A. 1979. Pragmatic connectives. Journal of Pragmatics, 3:447-456.

Webber, Bonnie L. 1988. Tense as discourse anaphor. Computational Linguistics, 14(2):61-72, June. 